\title{
Agonist-Induced Changes in Substituted Cysteine Accessibility Reveal Dynamic Extracellular Structure of M3-M4 Loop of Glutamate Receptor GluR6
}

\author{
Shahin S. Basiry, ${ }^{1}$ Paul Mendoza, ${ }^{1}$ Peter D. Lee, ${ }^{1}$ and Lynn A. Raymond ${ }^{1,2,3}$ \\ ${ }^{1}$ Kinsmen Laboratory of Neurological Research, Department of Psychiatry, ${ }^{2}$ Department of Physiology and ${ }^{3}$ Division of \\ Neurology, University of British Columbia, Vancouver, British Columbia V6T 1 Z3 Canada
}

Recent evidence suggests that the transmembrane topology of ionotropic glutamate receptors differs from other members of the ligand-gated ion channel superfamily. However, the structure of the segment linking membrane domains M3 and M4 (the M3-M4 loop) remains controversial. Although various data indicate that this loop is extracellular, other results suggest that serine residues in this segment are sites of phosphorylation and channel modulation by intracellular protein kinases. To reconcile these data, we hypothesized that the M3-M4 loop structure is dynamic and, more specifically, that the portion containing putative phosphorylation sites may be translocated across the membrane to the cytoplasmic side during agonist binding. To test this hypothesis, we mutated Ser 684, a putative cAMPdependent protein kinase site in the kainate-type glutamate receptor GluR6, to Cys. Results of biochemical and electrophysiological experiments are consistent with Cys 684 being accessible, in the unliganded state, from the extracellular side

Ionotropic glutamate receptors (GluRs) mediate most excitatory synaptic transmission and play an important role in triggering neuronal death (Coyle and Puttfarcken, 1993; Choi, 1994). Cloning and expression studies demonstrate that subclasses of GluRs are composed of homologous subunits: GluR1, $-2,-3$, and -4 for AMPA; GluR5, -6, and -7 and KA-1 and -2 for kainate (KA); and NR1 with NR2A, -B, -C, and -D for NMDA (Hollmann and Heinemann, 1994). A more detailed understanding of GluR molecular structure and topology is required, however, for the rational development of clinically useful, subtype-specific agents.

GluR transmembrane topology is unique among members of the ligand-gated ion channel superfamily. The pore-forming region, M2, forms a hairpin within the membrane (Hollmann et al., 1994; Stern-Bach et al., 1994; Wo and Oswald, 1994; Bennett and Dingledine, 1995; Wood et al., 1995; Kuner et al., 1996), and the C terminus is intracellular (Petralia and Wenthold, 1992; Tingley et al., 1993; Molnar et al., 1994). Furthermore, various data indicate that the M3-M4 loop is extracellular, because this region

Received Aug. 7, 1998; revised Oct. 30, 1998; accepted Nov. 2, 1998.

This work was supported by Medical Research Council (MRC, Canada) Operating Grant MT-12699 (L.A.R.). L.A.R. is an MRC Scholar. We thank T. H. Murphy, S. L. Slatin, R. Molday, and C. McIntosh for helpful discussions and advice. We are grateful to R. L. Huganir and C. A. Doherty for generously providing anti-GluR6/7 antibodies, to G. Kenner for technical support, and to S. Sturgeon for assistance in manuscript preparation.

Correspondence should be addressed to Dr. Lynn A. Raymond, Kinsmen Laboratory of Neurological Research, Department of Psychiatry, University of British Columbia, 4N3-2255 Wesbrook Mall, Vancouver, British Columbia, V6T 1 Z3 Canada.

Copyright (C) 1999 Society for Neuroscience $\quad 0270-6474 / 99 / 190644-09 \$ 05.00 / 0$ to modification by a Cys-specific biotinylating reagent followed by streptavidin (SA). Interestingly, our data suggest that this residue becomes inaccessible to the extracellular biotinylating reagent during agonist binding. However, we find it unlikely that Cys 684 undergoes membrane translocation, because the addition of SA to Cys-biotinylated GluR6(S684C) has no effect on peak glutamate-evoked current and only a small effect on macroscopic desensitization. We conclude that residue 684 in GluR6 is extracellular in the receptor-channel's closed, unliganded state and does not cross the membrane after agonist binding. However, an agonist-induced conformational change in the receptor substantially alters accessibility of position 684 to the extracellular environment.

Key words: kainate receptor; membrane topology; cysteine substitution; biotin; patch-clamp recording; human embryonic kidney 293 cells includes naturally glycosylated residues as well as amino acids involved in agonist binding or receptor desensitization (Sommer et al., 1990; Lomeli et al., 1994; Mosbacher et al., 1994; Roche et al., 1994; Taverna et al., 1994; Wo and Oswald, 1994; Paas et al., 1996; Partin et al., 1996; Swanson et al., 1997). Paradoxically, however, serine residues within the N-terminal half of the M3-M4 loop of AMPA/KA-type GluRs have been identified as cAMP-dependent protein kinase (PKA), $\mathrm{Ca}^{2+} /$ calmodulindependent protein kinase, or protein kinase $\mathrm{C}$ phosphorylation sites, suggesting an intracellular location for this region (McGlade-McCulloh et al., 1993; Raymond et al., 1993; Wang et al., 1993; Nakazawa et al., 1995; Yakel et al., 1995).

Two possible explanations for this conflicting data for the $\mathrm{N}$-terminal half of the AMPA/KA receptor M3-M4 loop are that (1) there are two additional transmembrane segments in this region, and (2) this structure is dynamic, lying extracellularly in the receptor's unliganded state but undergoing membrane translocation during agonist binding and channel gating. Both explanations seem energetically implausible, because there are no sustained segments of hydrophobic residues in this region (Asn 623 to approximately Thr 710 in GluR6). Moreover, models of GluRs based on the known structure of the highly homologous bacterial lysine/arginine/ornithine-binding protein LAOBP (Stern-Bach et al., 1994; Wo and Oswald, 1994; Sutcliffe et al., 1996) do not support the first explanation. In favor of the second explanation, gating-associated membrane translocation of polar and even charged residues has been demonstrated for some voltage-gated channels (Slatin et al., 1994; Larsson et al., 1996). 
Here, we tested the possibility that the putative target for PKA phosphorylation, Ser 684 of GluR6, undergoes membrane translocation during agonist binding. Cysteine-specific biotinylation of wild-type and mutant (S684C) GluR6 was compared using biochemical methods, and functional consequences were assessed by patch-clamp recording. Our results are consistent with an extracellular location for residue 684 in the channel's unliganded state. Moreover, although our data suggest that agonist-induced conformational changes significantly decrease accessibility of 684 to aqueous solution, they are inconsistent with membrane translocation of this residue.

\section{MATERIALS AND METHODS}

Construction of site mutation in GluR6. The cDNA encoding rat GluR6 (a gift from S. Heinemann, Salk Institute) was subcloned into a mammalian expression vector containing the cytomegalovirus promoter, as described previously (Raymond et al., 1993). Site-directed mutagenesis was performed using the Stratagene Chameleon Kit. To generate the Ser to Cys mutation at position 684 (S684C; amino acid numbering based on mature protein), we used 5'-CAG GAG ACA GTG TGT GCT TGT CAA AAG CAA TGA GG-3' as the mutagenesis primer and 5'-AGA GGA ACT TGG TTA GGG CCC TTC TGA GGC GGA AAG AAC-3' as the selection primer, converting a KpnI to an $A p a \mathrm{I}$ restriction site in the vector. The mutation was confirmed by restriction analysis and standard sequencing methods.

Transient transfection of wild-type and mutant GluR6 in human embryonic kidney 293 cells. Human embryonic kidney 293 (HEK 293) cells from American Type Culture Collection (CRL 1573) were cultured as described previously (Raymond et al., 1996). The cells were passaged every $2-3 \mathrm{~d}$ and plated at a density of $1-2 \times 10^{6}$ cells $/ \mathrm{ml} 10-14 \mathrm{hr}$ before transfection. Cells were plated directly onto poly-D-lysine $(10 \mu \mathrm{g} / \mathrm{ml})-$ coated culture dishes in preparation for biochemical experiments. The cells were transiently transfected with cDNA encoding $w t$ or mutant GluR6 (10 $\mu \mathrm{g}$ plasmid/10 cm plate) using calcium-phosphate coprecipitation, as described (Chen et al., 1997).

Biotinylation and Western blot analysis of wt and mutant GluR6. Biochemical analysis was performed $48 \mathrm{hr}$ after transfection. Transfected cells were washed twice with warm PBS and incubated at $37^{\circ} \mathrm{C}$ and $5 \%$ $\mathrm{CO}_{2}$ for 30-60 min with either $N$-hydroxysuccinimide-SS-biotin (NHSSS-biotin; $1 \mathrm{mg} / \mathrm{ml}$ ) or $N$-[6-(biotinamido)hexyl]-3'-(2'-pyridyldithio) propionamide (HPDP-biotin; $0.03 \mathrm{mg} / \mathrm{ml}$ ) in serum-free medium or in balanced saline solution (Hall et al., 1997). Cells were then washed five to six times with warm PBS to remove the biotinylating reagent. Isolation of the membrane fraction and precipitation of biotinylated proteins were performed essentially as described elsewhere (Hall et al., 1997). Briefly, the cells were collected in ice-cold harvest buffer containing $1 \mathrm{~mm}$ EDTA, $1 \mathrm{~mm}$ EGTA, $40 \mathrm{U} / \mathrm{ml}$ aprotonin (Trasylol), and $1 \mathrm{~mm}$ phenylmethylsulfonyl fluoride in PBS, $\mathrm{pH} 7.4$, lysed by $15 \mathrm{sec}$ sonication, and centrifuged for $20 \mathrm{~min}$ at $4^{\circ} \mathrm{C}, 14,000 \mathrm{rpm}$ (Eppendorf Microcentrifuge 5415C). Membrane proteins were isolated by resuspending the pellet in $1 \mathrm{ml}$ of harvest buffer containing $1 \%$ Triton X-100, centrifuging the suspension $\left(5 \mathrm{~min}, 5000 \mathrm{rpm}, 4^{\circ} \mathrm{C}\right)$, and collecting the supernatant. Aliquots of the membrane preparation, ranging from 10 to $80 \mu \mathrm{l}$ and corresponding to $1-8 \%$ of total protein, were reserved for loading on SDS-PAGE. The rest of the membrane preparation was incubated endover-end with $\sim 100 \mu \mathrm{l}$ streptavidin (SA)-linked beads at $4^{\circ} \mathrm{C}$ for $2 \mathrm{hr}$. Beads were collected by brief centrifugation and washed extensively with $1 \%$ Triton X-100 in harvest buffer. Bead-precipitated protein was eluted by end-over-end incubation in 100-200 $\mu \mathrm{l}$ gel loading buffer (containing $150 \mathrm{~mm}$ dithiothreitol) at $4^{\circ} \mathrm{C}$ for $30 \mathrm{~min}$ followed by brief centrifugation. The supernatant, together with the aliquots of the total membrane preparation, were subjected to $8 \%$ SDS-PAGE. Proteins were transferred to a polyvinylidene difluoride (PVDF) membrane, immunoblotted using affinity-purified anti-GluR6/7 polyclonal antibodies $(0.7 \mu \mathrm{g} / \mathrm{ml})$, and visualized using enhanced chemiluminescence (ECL). Protein bands were quantitated by densitometry, and a standard curve was constructed using measurements made from the lanes containing $1,2,4$, and $8 \%$ of the total membrane protein. This curve was used to quantitate the amount of bead-eluted GluR6, corresponding to biotinylated GluR6, as a percentage of total membrane protein.

Electrophysiology. Immediately after transfection, cells for patch-clamp recording were replated into $35 \mathrm{~mm}$ culture dishes containing glass coverslips. The cells were transferred on a glass coverslip to the stage of an inverted microscope (Axiovert 100, Carl Zeiss, Thornburg, NY) 48-80 hr after transfection. Patch-clamp recordings (Hamill et al., 1981) were made at room temperature $\left(20-22^{\circ} \mathrm{C}\right)$. Currents were sampled at 10 $\mathrm{KHz}$, filtered at $5 \mathrm{KHz}$, and acquired and analyzed using pCLAMP6 software and the Axopatch 200A amplifier (Axon Instruments, Foster City, CA).

Electrodes were fabricated from $1.5 \mathrm{~mm}$ outer diameter thin-wall borosilicate glass (Warner Instrument Corporation, Hamden, CT) using the Narishige PP-83 vertical puller (Narishige Scientific Instruments, Tokyo, Japan). Electrode resistance ranged from 2 to $5 \mathrm{M} \Omega$ when electrodes were filled with solution containing (in $\mathrm{mM}$ ): $145 \mathrm{KCl}, 5.5$ BAPTA, $0.5 \mathrm{CaCl}_{2}, 2 \mathrm{MgCl}_{2}, 2$ tetraethylammonium chloride, 4 MgATP, and 10 HEPES, pH 7.2.

Cells were continuously superfused with extracellular recording solution containing (in $\mathrm{mM}$ ): $145 \mathrm{NaCl}, 5.4 \mathrm{KCl}, 1.8 \mathrm{CaCl}_{2}, 1 \mathrm{MgCl}_{2}, 11$ glucose, and 10 HEPES, $\mathrm{pH}$ 7.35. Immediately after seal formation, each cell was lifted from the floor of the recording chamber and placed within $100 \mu \mathrm{m}$ of the tip of a theta tube (Hilgenberg, Malsfeld, Germany); each barrel tip had an inner diameter of $\sim 200 \mu \mathrm{m}$. Cells with membrane capacitance ranging from 8 to $16 \mathrm{pF}$ were chosen for recording. Control and agonist solutions were continuously gravity-fed through the two sides of the theta tube. Rapid exchange between these two solutions was accomplished by a computer-triggered piezo-electric device (Physik Instruments, Waldbronn, Germany), as described previously (Chen et al., 1997). The $10-90 \%$ rise time for exchange of the two solutions at the open tip of the recording electrode was $<0.5 \mathrm{msec}$. Agonist was applied for $100 \mathrm{msec}$ at $60 \mathrm{sec}$ intervals to monitor the peak current amplitude and desensitization time constant. Cells were exposed to $0.03 \mathrm{mg} / \mathrm{ml}$ HPDP-biotin (with or without $1 \mathrm{~mm}$ kainate) or $0.01 \mathrm{mg} / \mathrm{ml}$ purified streptavidin by continuous superfusion through the control side of the theta tube. Application of HPDP-biotin was begun only after a stable current response was established $(\sim 5-6 \mathrm{~min}$ after initial agonist application).

Data analysis. Current recordings were stored on hard disk for later analysis by pCLAMP6 Clampfit software, using a Pentium $90 \mathrm{MHz}$ personal computer. Desensitization time constants were determined by adjusting cursors in Clampfit to find the best (visual) fit of the current decay to a single exponential function, using the Chebyshev method; peak current was taken to be the amplitude extrapolated from that fit by the Clampfit program. Curve-fitting for dose-response data and the generation of standard curves from densitometry measurements were accomplished with Origin or Excel software, respectively. All values are shown as mean \pm SEM, unless indicated otherwise. Statistical comparisons were made using the two-tailed Student's $t$ test, either paired or unpaired (as specified), with a $95 \%$ confidence limit.

Materials. NHS-SS-biotin was dissolved into the experimental solution at $1 \mathrm{mg} / \mathrm{ml}$ just before use. HPDP-biotin was made up as a $3 \mathrm{mg} / \mathrm{ml}$ stock solution in dimethylsulfoxide (DMSO) and kept at $4^{\circ} \mathrm{C} ; 1 \mathrm{mg} / \mathrm{ml}$ streptavidin stock solutions were made up in extracellular recording solution on the day of use and kept on ice. For patch-clamp recording experiments, HPDP-biotin and streptavidin stocks were diluted 100-fold into extracellular recording solution just before addition to the cells. Stock solutions of kainate and glutamate $(100 \mathrm{~mm}$ and $1 \mathrm{M}$, respectively), as well as of CNQX (20 mM in DMSO), were maintained at $-20^{\circ} \mathrm{C}$ and thawed only once.

Streptavidin-linked beads, HPDP-biotin, and sulfo-NHS-SS-biotin were from Pierce (Rockford, Illinois). Purified streptavidin was from Molecular Probes (Eugene, Oregon). CNQX was from RBI (Natick, MA). Culture media and reagents were from Canadian Life Technologies (Burlington, ON). PVDF membranes and SDS-PAGE reagents were from Bio-Rad Laboratories (Hercules,CA). ECL reagents were from Amersham (Buckinghamshire, England). Anti-GluR6/7 polyclonal antibodies either were gifts from Dr. Richard Huganir (The Johns Hopkins University, Baltimore, MD) or were purchased from Upstate Biotechnology (Lake Placid, NY). All other reagents were from Sigma (St. Louis, MO).

\section{RESULTS}

\section{Increased cysteine-specific biotinylation of GluR6(S684C) compared with wt GluR6}

In a previous study, Slatin et al. (1994) used cysteine substitution followed by cysteine-specific biotinylation of the bacterial channel colicin IA to show that voltage-dependent transitions to the open or closed states could be prevented by the addition of streptavidin 


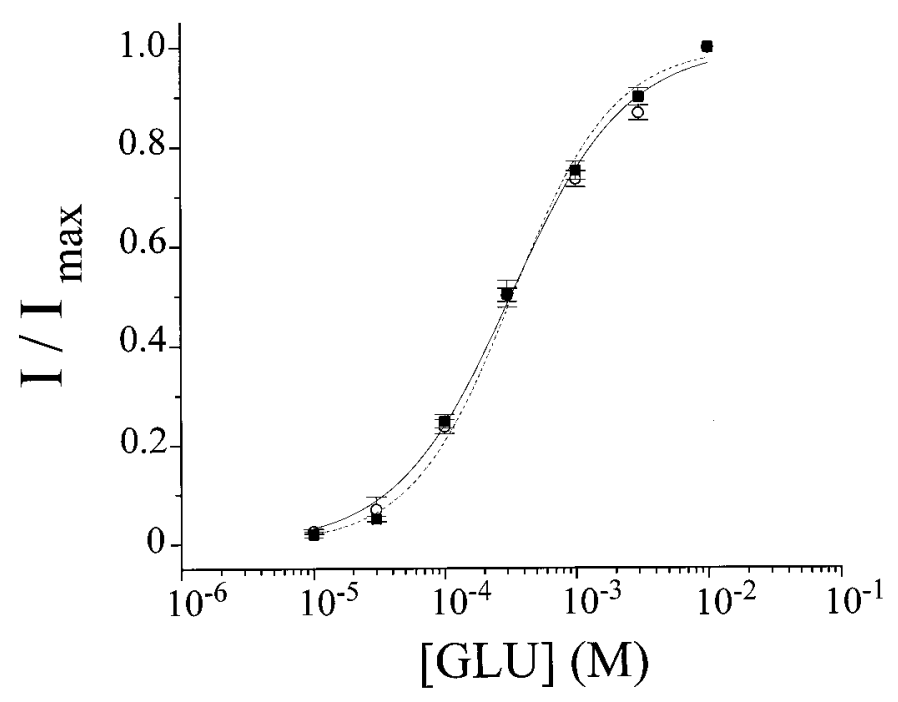

Figure 1. Glutamate dose-response curves for $w t$ GluR6 and GluR6(S684C) are similar. Peak glutamate-evoked current was recorded from cells transfected with either wt GluR6 (ם) or GluR6(S684C) (O), and the amplitude $(I)$ was normalized to the peak current response to 10 mM glutamate $\left(I_{\max }\right)$ for each cell. Points represent data from $n=3-9$ different cells. Curves were fitted to the equation $I=I_{\max }(1 /(1+$ $\left.\left(\mathrm{EC}_{50} /[\mathrm{GLU}]\right)^{n}{ }_{\mathrm{H}}\right)$ ), where $n_{\mathrm{H}}$ is the Hill coefficient. The $\mathrm{EC}_{50}$ and $n_{\mathrm{H}}$ values were $330 \pm 20 \mu \mathrm{M}$ and 1.1 for $w t$ GluR6, and $320 \pm 10 \mu \mathrm{M}$ and 1.0 for GluR6(S684C).

to the cis or trans side of a lipid bilayer, respectively. Therefore, to investigate the location of Ser 684 in the agonist bound and unbound state of GluR6, we first mutated this residue to Cys to permit modification of this site by Cys-specific biotinylating reagents and streptavidin. Importantly for our study, whole-cell patch-clamp recordings made from HEK 293 cells expressing the mutant GluR6(S684C) exhibited $1 \mathrm{~mm}$ glutamate (GLU)-evoked current responses similar to those recorded from wt GluR6transfected cells (see Fig. 5A). Furthermore, the GLU doseresponse curves for these two receptors were nearly identical (Fig. 1 ), as were other macroscopic properties of the GLU-evoked currents (Table 1).

To test whether Cys 684 is accessible to modification from the extracellular side of the cell membrane, we briefly incubated live GluR6(S684C)- or wt GluR6-transfected cells with the Cysspecific reagent HPDP-biotin (Slatin et al., 1994). Western blot analysis with GluR6-specific antibodies was used to compare the amount of biotinylated receptor recovered after incubation with SA-linked beads. In addition, to measure total wt GluR6 and GluR6(S684C) surface receptor expression, we used extracellular NHS-SS-biotin, which targets primary amines, so that all surface receptors would be expected to incorporate at least one biotin and thus be recovered by SA-linked bead precipitation. By the latter method, surface expression of $w t$ GluR6 and GluR6(S684C) was very similar: $4.8 \pm 0.3 \%(n=11)$ and $4.1 \pm 0.5 \%(n=7)$ of total receptor in the cell lysate, respectively (Fig. $2 A, D$ ). On the other hand, wt GluR6 recovered with SA-linked beads after incubation with HPDP-biotin was only $2.5 \pm 0.5 \%(n=11)$, or $\sim 50 \%$ of that recovered after incubation with NHS-SS-biotin (Fig. 2B,D) (significant difference by unpaired $t$ test, $p=0.0004$ ). Diminished recovery after HPDP-biotin is not surprising, because $w t$ GluR6 contains just seven putative extracellular Cys residues, and only those that are in the reduced state and relatively exposed to aqueous solution (i.e., not buried within a globular protein domain) are available for modification by HPDP-biotin. In contrast, SA-linked bead recovery of GluR6(S684C) after incubation with HPDP-biotin was $4.5 \pm$ $0.8 \%(n=12)$ (Fig. $2 B, D)$, which was significantly higher than that of $w t$ GluR6 ( $p=0.04$, unpaired $t$ test) and not significantly different from GluR6(S684C) recovery after treatment with NHS-SS-biotin ( $p=0.699$, unpaired $t$ test). As a control to confirm that extracellularly applied HPDP-biotin modifies only extracellular Cys residues, we used the same protocol to measure recovery of the cytoplasmic enzyme, microtubule-associated protein kinase (MAPK), by SA-linked bead precipitation. Western blot analysis with antibodies specific for MAPK showed no signal in the bead-precipitated fraction despite a robust signal for the cell lysate (data not shown), confirming that there was no significant biotinylation of MAPK by extracellularly applied HPDPbiotin. Taken together, these data suggest that Cys 684 is freely accessible to aqueous solution (and HPDP-biotin) on the extracellular side of the membrane.

\section{Decreased accessibility of Cys 684 to biotinylation after agonist binding}

To determine whether Cys 684 remains extracellular and accessible to modification when the receptor is in the ligand-bound state, we compared SA-linked bead recovery of wt GluR6 and GluR6(S684C) after incubation with HPDP-biotin in the presence versus absence of a saturating concentration of agonist. For $w t$ GluR6, there was a small increase in SA-linked bead recovery when $1 \mathrm{~mm}$ kainic acid (KA) was included during incubation with HPDP-biotin (Fig. $3 A, D)$, but this trend was not significant ( $n=$ $8 ; p=0.10$, paired $t$ test). In contrast, recovery of GluR6(S684C) decreased significantly ( $n=9 ; p=0.031$, paired $t$ test), to approximately the same level as seen for $w t$ GluR6, when HPDPbiotin incubation was performed in the presence of $1 \mathrm{mM} \mathrm{KA}$ (Fig. 3B,D). The difference between wt GluR6 and GluR6 (S684C) in the ratio of receptor recovered by SA-linked beads after incubation with HPDP-biotin in the presence of $1 \mathrm{~mm} \mathrm{KA}$ to that recovered under the control condition (HPDP-biotin incubation without KA) for each of the experiments was highly

Table 1. Comparison of macroscopic current properties of mutant and wt GluR6

\begin{tabular}{lllll} 
& $\tau_{\mathrm{D}}{ }^{a}(\mathrm{msec})$ & ${\text { Rise } \operatorname{time}^{b}(\mathrm{msec})}$ & $V_{\mathrm{rev}}{ }^{c}(\mathrm{mV})$ & $G_{+80} / G_{-80}{ }^{d}$ \\
\hline wt GluR6 & $6.0 \pm 0.2(54)$ & $1.9 \pm 0.06(35)$ & $1.3 \pm 1.3(9)$ & $1.5 \pm 0.2(14)$ \\
GluR6(S684C) & $6.1 \pm 0.1(51)$ & $2.0 \pm 0.05(29)$ & $1.2 \pm 1.4(10)$ & $1.8 \pm 0.3(10)$ \\
\hline
\end{tabular}

All measurements were made for current responses to rapid application of $1 \mathrm{~mm}$ glutamate for $100 \mathrm{msec}$.

${ }^{a}$ Desensitization time course was fit to a single exponential using CLAMPFIT software.

${ }^{b}$ Measured as time from 10 to $90 \%$ of peak current response.

${ }^{c}$ Holding potential was stepped from $-60 \mathrm{mV}$ to potentials ranging from -100 to $+100 \mathrm{mV}$ in $20 \mathrm{mV}$ increments every 20 sec before application of glutamate; peak current responses were measured.

${ }^{d}$ Ratio of conductance measured at $+80 \mathrm{mV}$ to that at $-80 \mathrm{mV}$. 

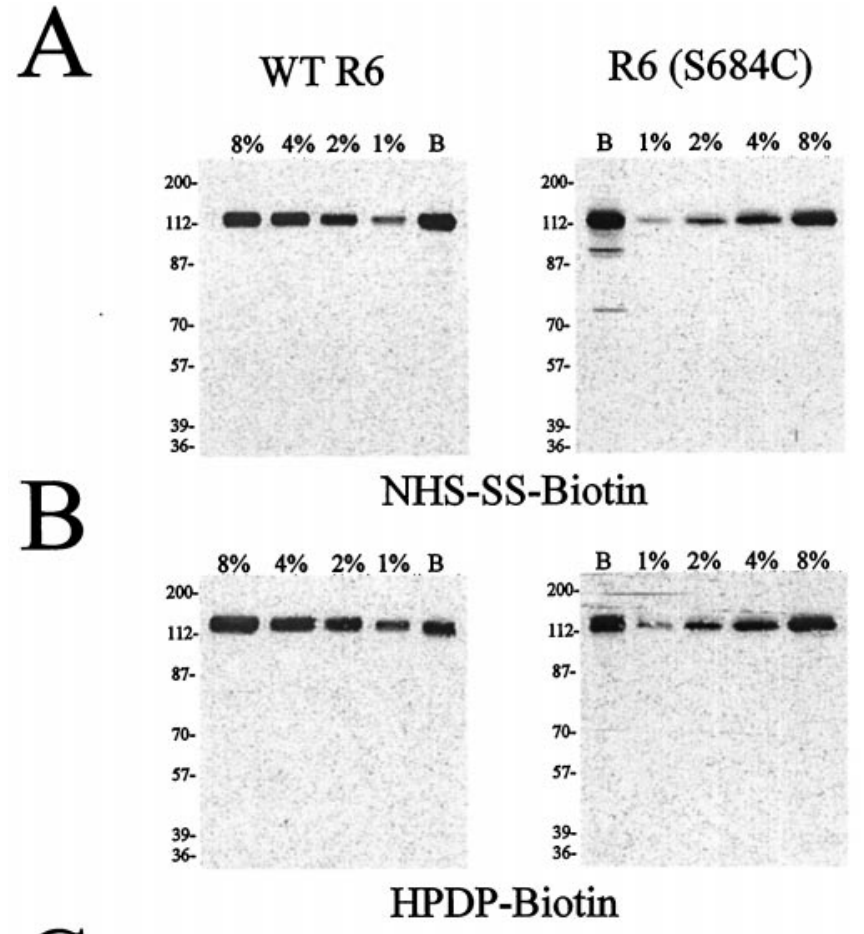

$\mathrm{C}$
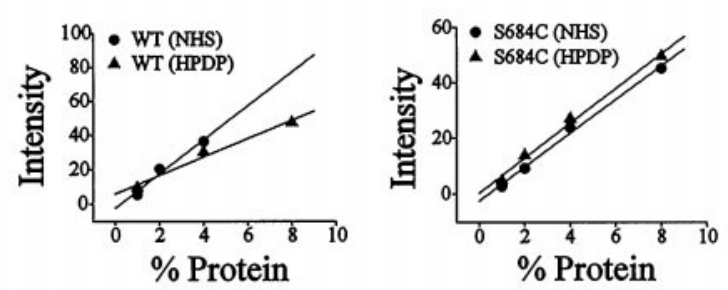

$\mathrm{D}$

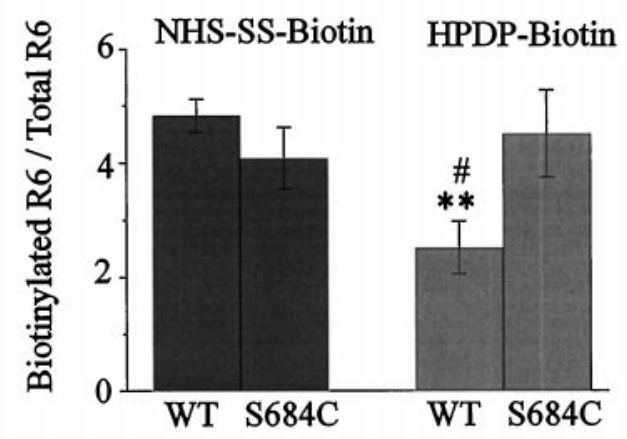

Figure 2. $\quad w t$ GluR6 shows significantly less cysteine-specific biotinylation than GluR6(S684C). $A, B$, Representative Western blot analyses of $w t$ GluR6 and GluR6(S684C) after incubation of transfected cells with NHS-SS-biotin ( $A$; primary amine-specific) or HPDP-biotin ( $B$; cysteinespecific), as described in Materials and Methods. $1,2,4$, and $8 \%$ represent the fraction of the total cell membrane isolate, and $B$ represents the total protein precipitated by streptavidin-linked beads, loaded in each lane. $C$, For blots shown in $A$ and $B$, band intensities were measured by densitometry from lanes containing $1,2,4$, and $8 \%$ of the total membrane protein to generate a standard curve. From these curves, the amount of biotinylated GluR6 was calculated as a fraction of the total membrane GluR6. D, Bars represent data from $n=7-12$ different experiments. \# Significant difference $(p<0.05)$ between wt GluR6 and GluR6(S684C) for biotinylation by HPDP-biotin; **significant difference $(p<0.001)$ for streptavidin bead recovery of $w t$ GluR6 after incubation with NHS-SS-biotin versus HPDP-biotin (both by unpaired $t$ test). significant ( $p=0.007$, unpaired $t$ test) (Fig. $3 D$ ). These data strongly suggest that the conformational change in GluR6(S684C) that is associated with KA binding renders Cys 684 inaccessible to modification by HPDP-biotin.

It is possible that mutation of Ser at position 684 results in a conformational change in the unliganded receptor, such that distant Cys residues become more accessible to aqueous solution (and HPDP-biotin), and that the conformation of ligand-bound GluR6(S684C) reverts to that of ligand-bound $w t$ GluR6. To test whether substitution of an amino acid other than Cys at position 684 results in comparable SA-linked bead recovery of receptors after HPDP-biotin versus NHS-SS-biotin, as well as significantly decreased SA-linked bead recovery after incubation with HPDPbiotin in the presence versus absence of agonist, we repeated the same experiments with GluR6(S684A). This mutant receptor also shows current responses and sensitivity to glutamate similar to $w t$ GluR6 (Raymond et al., 1993). Like wt GluR6, we found that SA-linked bead recovery of GluR6(S684A) after incubation with HPDP-biotin was markedly lower than that recovered after treatment with NHS-SS-biotin (ratio of $0.57 \pm 0.12, n=3$ ). Moreover, SA-linked bead recovery of GluR6(S684A) after HPDP-biotin incubation in the presence versus absence of $1 \mathrm{~mm}$ kainate was not significantly different (ratio of $1.01 \pm 0.06, n=4 ; p=0.656$, paired $t$ test), again similar to that observed for $w t$ GluR6. These data indicate that mutation of Ser 684 alone (i.e., to an amino acid other than Cys) is not sufficient to alter Cys-specific biotinylation of the receptor. These results, together with the fact that GluR6(S684C) current responses and sensitivity to glutamate are similar to that of $w t$ GluR6, support the conclusion that the substituted Cys at 684 is biotinylated by HPDP-biotin and that accessibility of this reagent to Cys 684 is markedly decreased in the presence of kainate.

\section{Agonist-induced conformational change alters accessibility of Cys 684 to extracellular reagent}

The decrease in accessibility of Cys 684 to extracellular HPDPbiotin observed in the presence of $1 \mathrm{mM}$ KA could be caused by (1) agonist-induced movement of residue 684 to a position less exposed to aqueous solution on the extracellular side of the membrane, (2) agonist-induced membrane translocation of this residue, or (3) the fact that occupation of the KA-binding site itself blocks access to Cys 684. Previous studies have indicated that different agonists and competitive antagonists coordinate with different amino acids within the binding pocket of GluRs (Paas et al., 1996; Swanson et al., 1997). Therefore, to test the third possibility, we compared SA-linked bead recovery of GluR6(S684C) after HPDP-biotin incubation in the presence versus absence of another agonist, glutamate, or the competitive antagonist CNQX (Honoré et al., 1988). Results for HPDP-biotin incubation in the presence versus absence of $1 \mathrm{~mm}$ glutamate are shown in Figure $4 A, D$. Similar to results of experiments with kainate, there was a marked decrease in Cys-specific biotinylation of GluR6(S684C) but little change in that of wt GluR6 when HPDP-biotin incubation was performed in the presence of $1 \mathrm{~mm}$ glutamate [significant difference between ratios for $w t$ and mutant GluR6 by unpaired $t$ test, $p=0.022$ (compare Figs. $3 D$ and $4 D$ )]. On the other hand, in the presence of a nearly saturating concentration of CNQX $(10 \mu \mathrm{M})$ (Wilding and Huettner, 1996), there was little change in HPDP-biotinylation of GluR6(S684C) compared with the absence of CNQX (Fig. $4 B, D$ ), and the ratio of recovery of biotinylated GluR6(S684C) in the presence versus absence of CNQX was significantly different from the ratio in the 


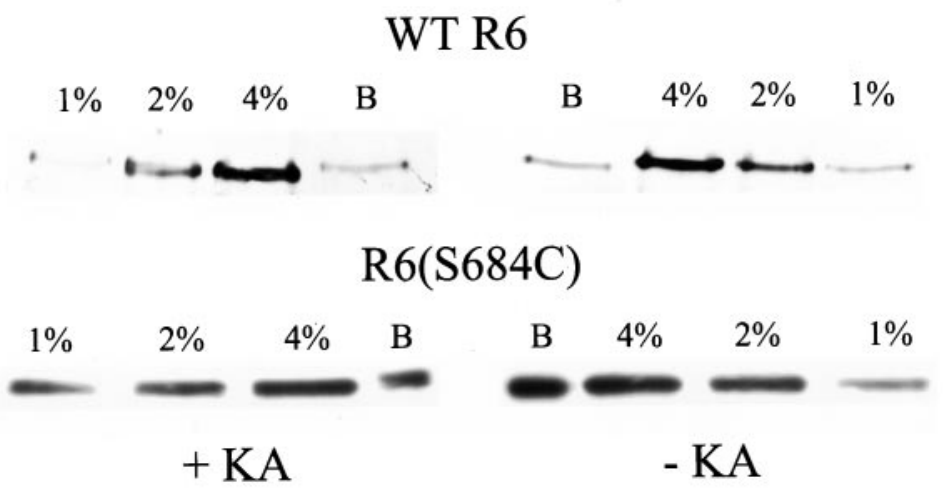

Figure 3. Cys-specific biotinylation of GluR6(S684C) shows significant decrease with kainate binding. $A, B$, Representative Western blot analyses of $w t$ GluR6 $(A)$ and GluR6(S684C) $(B)$ after incubation of transfected cells with HPDP-biotin in the presence $(+K A)$ versus absence $(-K A)$ of $1 \mathrm{~mm}$ kainate. Left and right panels are from same gel. Labeling of lanes is as described in Figure 2. $C$, Standard curves were generated from blots shown in $A$ and $B$, as described in Figure $2 C$, and such curves were used to determine the amount of Cys-biotinylated GluR6. D, Ratio of Cys-biotinylated GluR6 in the presence versus absence of kainate was calculated for each of $n=8(W T R 6)$ or $n=$ $9[R 6(S 684 C)]$ different experiments. ${ }^{*}$ Significant difference ( $p<0.01$ by unpaired $t$ test) between $w t$ GluR6 and GluR6(S684C).
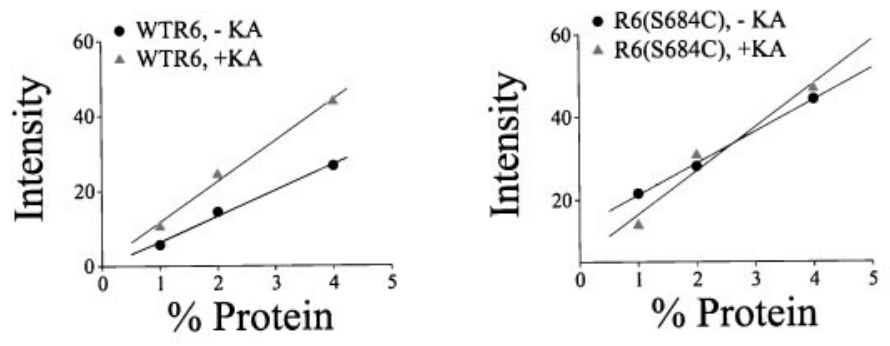

$\mathrm{D}$

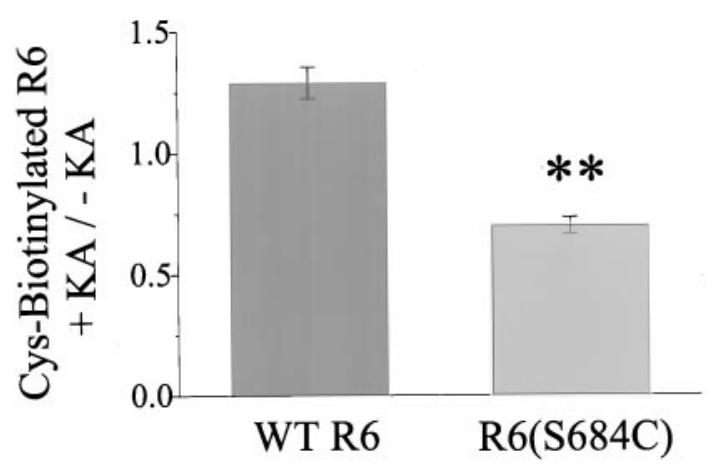

presence versus absence of glutamate or kainate $(p=0.016$ and 0.027 , respectively, unpaired $t$ test). Apparently, both occupation of the ligand binding site and channel gating are required for Cys 684 to become inaccessible to extracellular HPDP-biotin.

\section{Cys-specific biotinylation alters peak current amplitude and desensitization of both wt and mutant GluR6}

To determine whether Cys 684 in GluR6(S684C) remains extracellular (although buried), or whether it is actually translocated to the cytoplasmic side of the membrane during agonist binding and channel activation, we performed whole-cell patch-clamp recording. We compared the current response evoked by $1 \mathrm{~mm}$ glutamate recorded from GluR6(S684C)- versus wt GluR6-transfected cells after incubation with HPDP-biotin followed by streptavidin. We assumed that any differences observed in the current response mediated by GluR6(S684C) compared with that of wt GluR6 would be caused by modification of Cys 684 .

As illustrated in Figure 5, incubation for $\sim 15$ min with HPDPbiotin, followed by 6-8 min of washout, had a similar effect on GluR6(S684C)- and wt GluR6-mediated currents. In both cases, we observed a significant, irreversible decrease of $1 \mathrm{~mm}$

glutamate-evoked peak current amplitude along with slowing of onset of agonist-induced desensitization $\left(\tau_{\mathrm{D}}\right)$, although the latter was not significant because of high variability in the extent of slowing. In contrast, a $15 \mathrm{~min}$ incubation with vehicle alone $(1 \%$ DMSO) resulted in a smaller decrease in peak current amplitude and no change in $\tau_{\mathrm{D}}$ (Fig. $5 B, C$ ). Thus, these alterations in macroscopic current would be consistent with effects of biotinylation of Cys residue(s) present in both $w t$ GluR6 and GluR6(S684C). From these results, we concluded that if Cys 684 was modified by HPDP-biotin under conditions used in these patch-clamp recording experiments, there was no functional effect of the addition of biotin to this residue. As a further test of this conclusion, we analyzed the effect of incubating GluR6(S684C)-transfected cells with HPDP-biotin in the presence of $1 \mathrm{~mm}$ kainate. As discussed above, analysis by Western blot suggested that inclusion of $1 \mathrm{~mm}$ kainate along with HPDPbiotin prevented biotinylation of Cys 684 (Fig. 3). Consistent with the conclusion that biotinylation of Cys 684 is functionally "silent," we found no significant difference in effects on GluR6(S684C) peak current amplitude or $\tau_{\mathrm{D}}$ after incubation with HPDP-biotin in the presence versus absence of $1 \mathrm{~mm}$ kainate (Fig. 5B,C). 
A

\section{R6(S684C)}

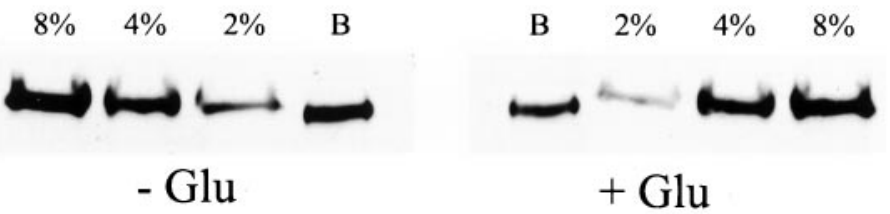

B
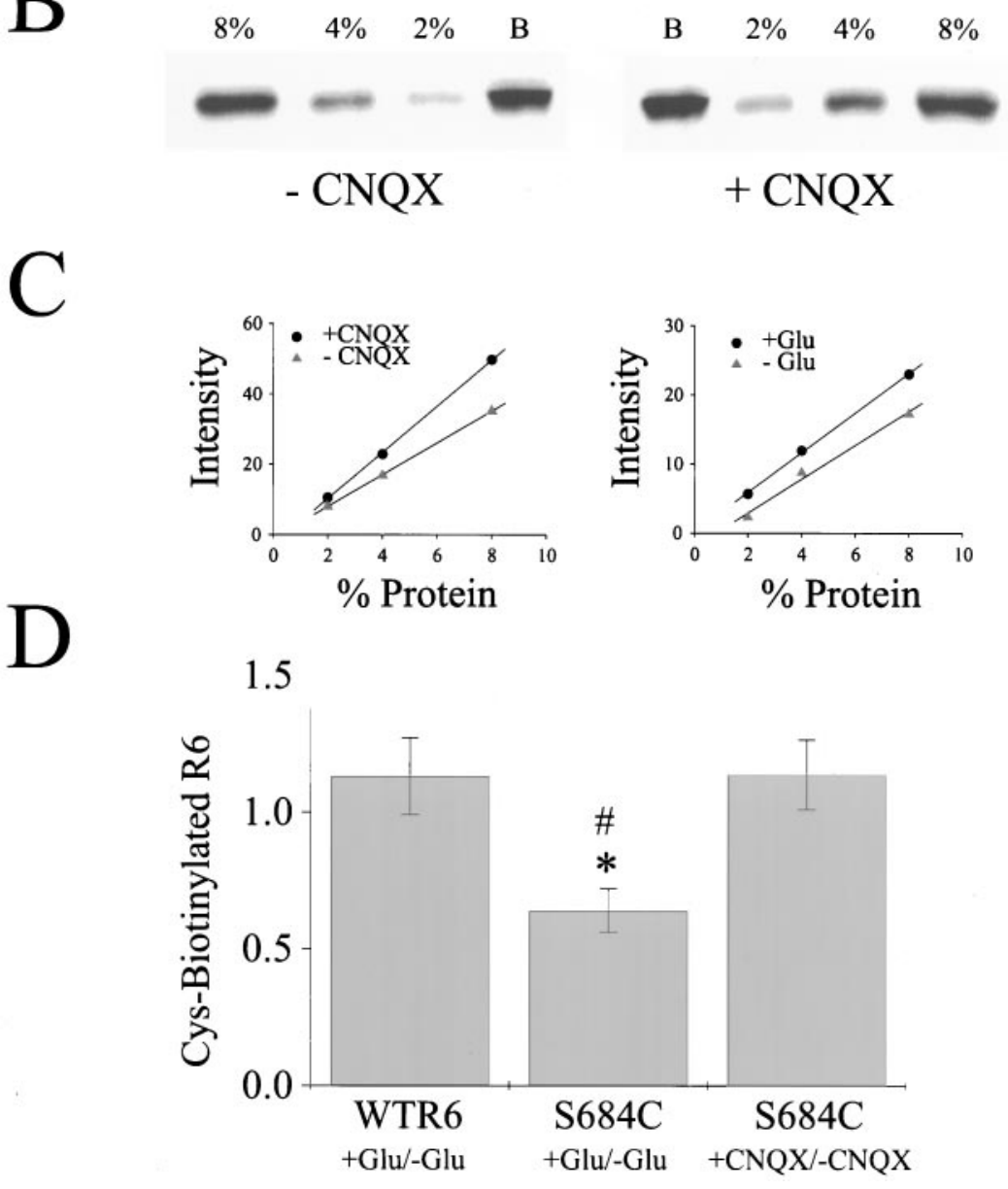

Figure 4. Occupation of ligand binding site and channel activation required to render Cys 684 inaccessible to HPDP-biotin. $A$, Binding of another agonist, glutamate, decreases Cys-specific biotinylation of GluR6(S684C). Representative Western blot analysis of GluR6(S684C)transfected cells treated with HPDP-biotin in the absence $(-G l u)$ versus presence $(+G l u)$ of $1 \mathrm{~mm}$ glutamate. $B$, Antagonist binding does not alter Cys-specific biotinylation of GluR6(S684C). Representative Western blot analysis of GluR6(S684C)-transfected cells treated with HPDP-biotin in the absence versus presence of $10 \mu \mathrm{M}$ CNQX. For both $A$ and $B$, left and right panels are from same gel. Labeling of lanes is as described in Figure 2. $C$, Standard curves were generated from blots shown in $A$ and $B$, as described in Figure 2C,D. Ratio of Cys-biotinylated GluR6 in the presence versus absence of Glu or CNQX was calculated from $n=4$ different experiments for each condition: WT R6, + Glu/-Glu; S684C, + Glu/-Glu; and S684C, +CNQX/-CNQX. *Significant difference $(p<$ 0.05 by unpaired $t$ test) between $w t$ GluR6 and GluR6(S684C) for +Glu/-Glu; * significant difference $(p<0.05$, unpaired $t$ test $)$ between $+\mathrm{Glu} /-$ Glu and $+\mathrm{CNQX} /-\mathrm{CNQX}$ for GluR6(S684C).

\section{Extracellular streptavidin treatment of Cys-biotinylated GluR6(S684C) leaves channel activation intact but slows desensitization}

Next we analyzed recordings made from $w t$ GluR6- and GluR6(S684C)-transfected cells after biotinylation and extensive washout of HPDP-biotin and then incubation with extracellular streptavidin. In contrast to HPDP-biotin, the addition of streptavidin had no significant effect on peak current amplitude for either wt GluR6 or GluR6(S684C) (Fig. 6A,B). On the other hand, there was a further significant slowing of onset of agonistinduced desensitization seen for GluR6(S684C)-mediated, but not $w t$ GluR6-mediated, currents after treatment with streptavidin (Fig. 6A,C). Moreover, if $1 \mathrm{~mm}$ kainate was included during the incubation with HPDP-biotin, streptavidin had no effect on the rate of macroscopic desensitization of GluR6(S684C)mediated current (Fig. 6A,C). Because an effect of streptavidin was seen for GluR6(S684C) and not for wt GluR6 after incubation with HPDP-biotin in the absence of agonist, we conclude that Cys 684 in GluR6(S684C) is biotinylated by HPDP-biotin and accessible to streptavidin from the extracellular side of the membrane, a result consistent with our biochemical evidence (see above). However, because streptavidin had no effect on peak current amplitude and only a small effect on macroscopic desensitization, it is unlikely that Cys 684 undergoes membrane translocation during agonist binding and channel activation. Taken together, the results of both biochemical and patch-clamp recording experiments suggest that the amino acid residue at position 684 in GluR6 is extracellular in the receptor's unliganded state as well as in the ligand-bound, activated state.

\section{DISCUSSION}

Using a combined biochemical and electrophysiological approach to analyze wt GluR6 and GluR6(S684C) after incubation with reagents that add biotin molecule(s) to cell surface proteins, we have shown data consistent with an extracellular location for amino acid 684 , in both the presence and absence of agonist. 
A
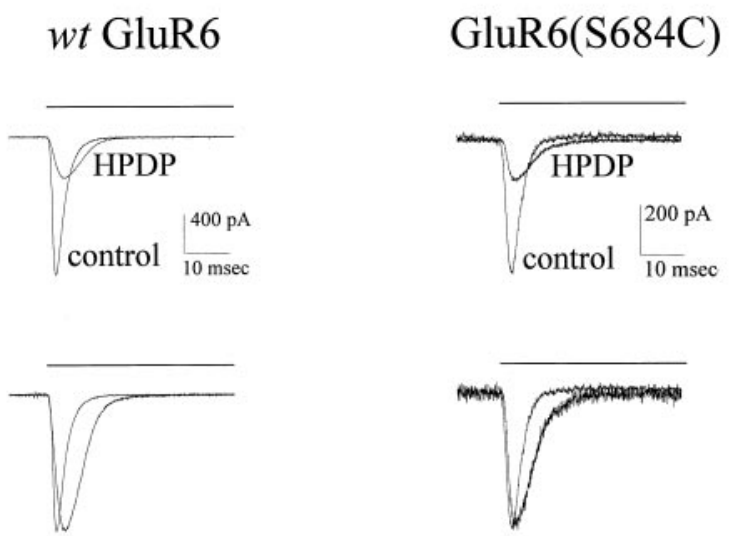

B
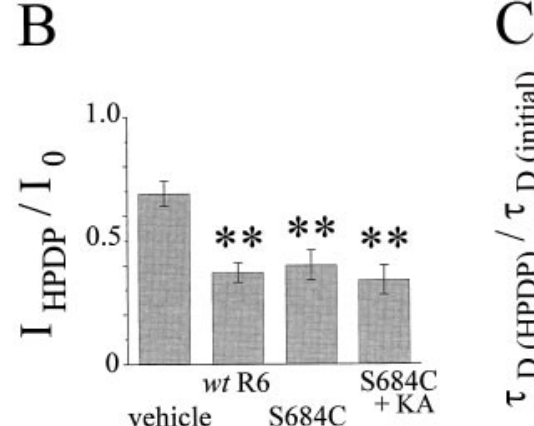

Figure 5. Cys-specific biotinylation decreases peak current amplitude and slows desensitization for both $w t$ GluR6 and GluR6(S684C). Wholecell patch-clamp recordings were made under voltage clamp $\left(V_{\mathrm{H}}=-60\right.$ $\mathrm{mV}$ ) from cells transfected with either wt GluR6 or GluR6(S684C). $A$, Top, Representative current responses to rapid application of $1 \mathrm{~mm}$ glutamate (indicated by bar) before (control) and after (HPDP) a $15 \mathrm{~min}$ incubation with $0.03 \mathrm{mg} / \mathrm{ml}$ extracellular HPDP-biotin followed by a 6-8 min washout period. A, Bottom, The gain of each HPDP trace has been increased to match the peak current amplitude of the corresponding control trace to better illustrate the slowing of desensitization. $B$, Peak current amplitude after 15 min incubation (and 6-8 min washout) with $1 \%$ DMSO (vehicle), $0.03 \mathrm{mg} / \mathrm{ml}$ HPDP-biotin (in $1 \% \mathrm{DMSO}$ ), or 0.03 $\mathrm{mg} / \mathrm{ml}$ HPDP-biotin in the continuous presence of $1 \mathrm{~mm} \mathrm{KA}(+K A)$, was normalized to the pretreatment peak current amplitude $\left(I_{0}\right)$. Bars represent data from $n=5$ different cells for vehicle [results from $w t$ GluR6- and GluR6 (S684C)-transfected cells were pooled], $n=11$ for $w t \mathrm{R} 6, n=9$ for S684C, or $n=8$ for S684C + KA. **Significant difference between vehicle and HPDP-biotin-treated groups by unpaired $t$ test, $p<0.01$. $C$, Desensitization time constant $\left(\tau_{\mathrm{D}}\right)$ after treatment with HPDP-biotin (as above) was normalized to pretreatment value $\left(\tau_{\mathrm{D} \text { (initial) }}\right)$. Bars represent data from $n=5$ (vehicle), 11 (wt R6), $9(\mathrm{~S} 684 \mathrm{C})$, or $8(\mathrm{~S} 684 \mathrm{C}+K A)$ different cells.

Interestingly, our results also suggest that an agonist-induced conformational change in GluR6 results in a major shift in the accessibility of this amino acid to aqueous extracellular solution.

In our biochemical analyses of biotinylated GluR6, we first used NHS-SS-biotin to determine the percentage of total membrane GluR6 expressed at the cell surface. Because this reagent targets primary amines, every surface receptor should receive multiple biotins and thus be isolated by streptavidin-linked bead precipitation. On the other hand, the addition of one biotin molecule per receptor should be sufficient to recover all surface receptors by streptavidin-linked bead precipitation. Our data showed complete recovery of surface GluR6(S684C) but only $\sim 50 \%$ recovery of surface $w t$ GluR6 after HPDP-biotin as compared with NHS-SSbiotin treatment. These results suggest that the cysteine substi-
A

\section{wt GluR6}

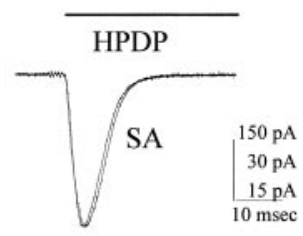

B

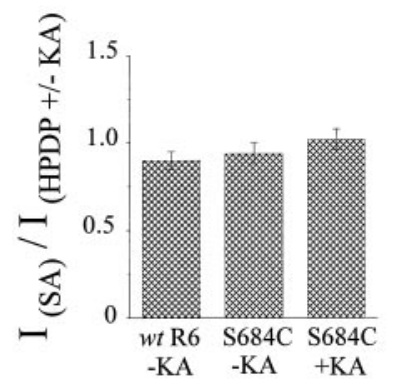

GluR6(S684C)

GluR6(S684C)
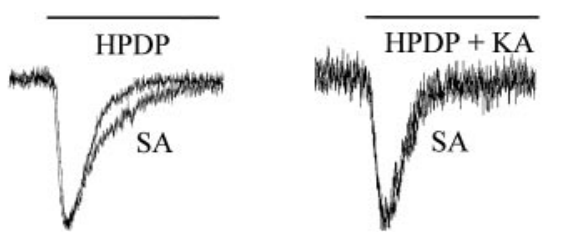

C
Figure 6. Extracellular streptavidin slows desensitization of Cysbiotinylated GluR6(S684C). Whole-cell patch-clamp recordings were made from cells expressing wt GluR6 or GluR6(S684C), as in Figure 5. A, Representative current responses to $1 \mathrm{mM}$ glutamate recorded from three different cells after HPDP-biotin treatment and washout period (HPDP; as in Fig. 5), and then 10-15 min incubation with $0.01 \mathrm{mg} / \mathrm{ml}$ streptavidin followed by $6-8 \mathrm{~min}$ washout $(S A)$. In far right panel, the cell was treated continuously with $1 \mathrm{~mm}$ kainate during the time of incubation with HPDP-biotin $(H P D P+K A) \cdot B$, Peak current amplitude after streptavidin treatment and washout $\left(I_{(\mathrm{SA})}\right)$ was normalized to peak current amplitude immediately after HPDP-biotin treatment (with or without $1 \mathrm{~mm}$ kainate) and washout period $\left(I_{(\mathrm{HPDP} \pm \mathrm{KA})}\right) . C$, Desensitization time constant measured after incubation with streptavidin was normalized to that measured immediately before streptavidin treatment (as in $B$ ). In both $B$ and $C$, bars represent data from $n=5$ for $w t \mathrm{R} 6,-\mathrm{KA} ; n=6$ for $\mathrm{S} 684 \mathrm{C},-\mathrm{KA}$; or $n=$ 5 for S684C, + 1 mM KA. *Significant difference between groups by unpaired $t$ test, $p=0.02$.

tuted at position 684 within the M3-M4 loop is in the reduced state and freely accessible to extracellular reagents, whereas the reduced cysteine(s) present in wt GluR6 are less accessible to these reagents.

For GluR6(S684C) we have demonstrated that accessibility of Cys 684 to Cys-specific biotinylating reagents is significantly decreased in the presence of agonist. Two different results support this conclusion: (1) decreased recovery of surface receptors by streptavidin-linked bead precipitation after Cys-specific biotinylation in the presence versus absence of agonist and (2) lack of effect of extracellular streptavidin on channel function after Cysspecific biotinylation in the presence of agonist. We have also shown that occupation of the ligand binding site is not sufficient to alter accessibility of Cys 684, because the competitive antagonist CNQX has no effect on the efficiency of biotinylation of Cys 684. Thus, we conclude that the shift in accessibility of Cys 684 is caused by an agonist-induced conformational change involved in channel activation and/or desensitization. Cys 684 may become "buried" in the protein milieu and thereby sequestered from aqueous extracellular solution. Our data do not support membrane translocation of Cys 684, because treatment of Cysbiotinylated GluR6(S684C) with extracellular streptavidin only subtly alters channel function. Moreover, the fact that 
GluR6(S684C) forms channels with characteristics essentially identical to $w t$ GluR6 suggests that the mutant receptor is normally folded and that these results may be generalized to position 684 of $w t$ GluR6.

Our results are in agreement with a model recently proposed by Swanson et al. (1997). In this model, position 684 ( 715 by their numbering scheme) is not itself exposed to the agonist binding cleft but is shown within a 32 amino acid region that is flanked by two amino acids involved in agonist binding and essentially lacks secondary structure, potentially allowing full exposure to aqueous solution in the unliganded state. Oh et al. (1993) proposed that ligand binding to the homologous protein LAOBP results in stabilization of a conformation in which lobes I and II are brought into close contact via rotation of lobe II around a hinge region. Because lobe II includes the segment of the M3-M4 loop containing amino acid 684 of GluR6, perhaps this residue is rotated from a position that is fully exposed to the aqueous environment to one that is in close contact with protein.

It is interesting that in our patch-clamp recording experiments, biotinylation of Cys residue(s) present in both mutant and wt GluR6 resulted in a decrease in peak current amplitude as well as slowing of desensitization, but subsequent treatment with streptavidin did not alter channel function further. However, results of our biochemical analysis indicate that Cys-specific biotinylation of $w t$ GluR6 is incomplete, likely because of low accessibility of reduced cysteine(s) to extracellular HPDP-biotin. Because streptavidin is a much larger molecule than HPDP-biotin, perhaps its access to this site is extremely limited. In contrast, biotinylation of Cys 684 appeared to be functionally silent, but subsequent streptavidin treatment of GluR6(S684C) resulted in a further slowing of desensitization. It is not surprising that the addition of such a large molecule at that site would interfere with desensitization, because other segments of the M3-M4 loop have been implicated in regulating desensitization of AMPA/KA receptors (Sommer et al., 1990; Lomeli et al., 1994; Mosbacher et al., 1994; Partin et al., 1996; Swanson et al., 1997).

Our data are consistent with an entirely extracellular M3-M4 loop, in agreement with the results of various other experimental approaches to determining GluR membrane topology (see introductory remarks). Moreover, a dynamic M3-M4 loop topology, in which position 684 and the surrounding region of GluR6 is translocated across the membrane during agonist binding and channel gating, is highly unlikely on the basis of our results. Nakazawa et al. (1995) raised the possibility of such a dynamic M3-M4 loop structure based on their data showing agonistdependent phosphorylation of Ser 696 in the AMPA subunit GluR2, but other interpretations for their results were also offered. As well, it is possible that membrane topology may vary between subunits. On the other hand, data from previous studies of GluR6, indicating that a Ser to Ala mutation at position 684 of GluR6 significantly decreased (Wang et al., 1993) or even eliminated (Raymond et al., 1993) the potentiating effect of intracellular PKA on whole-cell current amplitude, remain puzzling. It is clear from a recent study, however, that intracellular perfusion with activated PKA during patch-clamp recording increases the channel open probability for GluR6 by nearly $50 \%$, from $P_{\text {open }}$ of $\sim 0.6$ to $\sim 0.9$ (Traynelis and Wahl, 1997). Possibly, Ser 684 is critical to channel gating, and if so, perhaps the Ser to Ala mutation results in a similar $50 \%$ increase in $P_{\text {open }}$, thereby occluding the effect of intracellular PKA. Further experiments are required to examine this issue.

In summary, our results are in agreement with the currently favored membrane topology model for GluRs, in which the M3-M4 loop is entirely extracellular. Moreover, at least for GluR6, our data do not support membrane translocation of putative phosphorylation sites within a portion of the M3-M4 loop. However, the position 684 within this loop appears to be involved in an agonist-induced conformational change associated with channel gating. These results provide further insight into the structure and membrane topology of the kainate receptor GluR6, information that may be useful for the future development of more potent specific agonists and antagonists for GluRs.

\section{REFERENCES}

Bennett JA, Dingledine R (1995) Topology profile for a glutamate receptor: three transmembrane domains and a channel-lining reentrant membrane loop. Neuron 14:3731-3784.

Chen N, Moshaver A, Raymond LA (1997) Differential sensitivity of recombinant $N$-methyl-D-aspartate receptor subtypes to zinc inhibition. Mol Pharmacol 51:1015-1023.

Choi DW (1994) Glutamate receptors and the induction of excitotoxic neuronal death. Prog Brain Res 100:47-51.

Coyle JT, Puttfarcken P (1993) Oxidative stress, glutamate, and neurodegenerative disorders. Science 262:689-695.

Hall RA, Hansen A, Andersen PH, Soderling TR (1997) Surface expression of the AMPA receptor subunits GluR1, GluR2, and GluR4 in stably transfected baby hamster kidney cells. J Neurochem 68:625-630.

Hamill OP, Marty A, Neher E, Sakmann B, Sigworth FJ (1981) Improved patch-clamp techniques for high resolution current recording from cells and cell free membrane patches. Pflügers Arch Eur J Physiol 391:85-100.

Hollmann M, Heinemann S (1994) Cloned glutamate receptors. Annu Rev Neurosci 17:31-108.

Hollmann M, Maron C, Heinemann S (1994) N-glycosylation site tagging suggests a three transmembrane domain topology for the glutamate receptor GluR1. Neuron 13:1331-1343.

Honoré T, Davies SN, Drejer J, Fletcher EJ, Jacobsen P, Lodge D, Nielsen FE (1988) Quinoxalinediones: potent competitive nonNMDA glutamate receptor antagonists. Science 241:701-703.

Kuner T, Wollmuth LP, Karlin A, Seeburg PH, Sakmann B (1996) Structure of the NMDA receptor channel M2 segment inferred from the accessibility of substituted cysteines. Neuron 17:343-352.

Larsson HP, Baker OS, Dalvinder, Dhillon DS, Isacoff EY (1996) Transmembrane movement of the shaker $\mathrm{K}^{+}$channel S4. Neuron 16:387-397.

Lomeli H, Mosbacher J, Melcher T, Höger T, Geiger JRP, Kuner T, Monyer H, Higuchi M, Bach A, Seeburg P (1994) Control of kinetic properties of AMPA receptor channels by nuclear RNA editing. Science 266:1709-1713.

McGlade-McCulloh E, Yamamoto H, Tan S-E, Brickey DA, Soderling TR (1993) Phosphorylation and regulation of glutamate receptors by calcium/calmodulin-dependent protein kinase II. Nature 362:640-642.

Molnar E, McIlhinney RAJ, Baude A, Nusser Z, Somogyi P (1994) Membrane topology of the GluR1 glutamate receptor subunit: epitope mapping by site-directed antipeptide antibodies. J Neurochem 63:683-693.

Mosbacher J, Schoepfer R, Monyer H, Burnashev N, Seeburg PH, Ruppersberg JP (1994) A molecular determinant for submillisecond desensitization in glutamate receptors. Science 266:1059-1062.

Nakazawa K, Mikawa S, Hashikawa T, Ito M (1995) Transient and persistent phosphorylation of AMPA-type glutamate receptor subunits in cerebellar Purkinje cells. Neuron 15:697-709.

Oh B-H, Pandit J, Kang C-H, Nikaido K, Gokcen S, Ferro-Luzzi Ames G, Kim S-H (1993) Three-dimensional structures of the periplasmic lysine/arginine/ornithine-binding protein with and without a ligand. J Biol Chem 268:11348-11355.

Paas Y, Eisenstein M, Medevielle F, Teichberg V, Devillers-Thiery A (1996) Identification of the amino acid subsets accounting for the ligand binding specificity of a glutamate receptor. Neuron 17:979-990.

Partin KM, Fleck MW, Mayer ML (1996) AMPA receptor flip/flop mutants affecting deactivation, desensitization, and modulation by cyclothiazide, aniracetam, and thiocyanate. J Neurosci 16:6634-6647.

Petralia RS, Wenthold RJ (1992) Light and electron immunocytochemical localization of AMPA-selective glutamate receptors in the rat brain. J Comp Neurol 318:329-354. 
Raymond LA, Blackstone CD, Huganir RL (1993) Phosphorylation and modulation of recombinant GluR6 glutamate receptors by cAMPdependent protein kinase. Nature 361:637-641.

Raymond LA, Moshaver A, Tingley WG, Shalaby I, Huganir RL (1996) Glutamate receptor ion channel properties predict vulnerability to cytotoxicity in a transfected nonneuronal cell line. Mol Cell Neurosci 7:102-115.

Roche KW, Raymond LA, Blackstone C, Huganir RL (1994) Transmembrane topology of the glutamate receptor subunit GluR6. J Biol Chem 269:11679-11682.

Slatin SL, Qiu X, Jakes KS, Finkelstein A (1994) Identification of a translocated protein segment in a voltage-dependent channel. Nature $371: 158-161$

Sommer B, Keinänen K, Verdoorn TA, Wisden W, Burnashev N, Herb A, Köhler M, Takagi T, Sakmann B, Seeburg PH (1990) Flip and flop: a cell specific functional switch in glutamate-operated channels of the CNS. Science 249:1580-1585.

Stern-Bach Y, Bettler B, Hartley M, Sheppard PO, O'Hara PJ, Heinemann SF (1994) Agonist selectivity of glutamate receptors is specified by two domains structurally related to bacterial amino acid-binding proteins. Neuron 13:1345-1357.

Sutcliffe MJ, Wo ZG, Oswald RE (1996) Three-dimensional models of non-NMDA glutamate receptors. Biophys J 70:1575-1589.

Swanson GT, Gereau IV RW, Green T, Heinemann SF (1997) Identification of amino acid residues that control functional behavior in GluR5 and GluR6 kainate receptors. Neuron 19:913-926.
Taverna FA, Wang L-Y, MacDonald JF, Hampson DR (1994) A transmembrane model for an ionotropic glutamate receptor predicted on the basis of the location of asparagine-linked oligosaccharides. J Biol Chem 269:14159-14164.

Tingley WG, Roche KW, Thompson AK, Huganir RL (1993) Regulation of NMDA receptor phosphorylation by alternative splicing of the C-terminal domain. Nature 364:70-73.

Traynelis SF, Wahl P (1997) Control of rat GluR6 glutamate receptor open probability by protein kinase A and calcineurin. J Physiol (Lond) 503:513-531.

Wang L-Y, Taverna FA, Huang X-P, MacDonald JF, Hampson DR (1993) Phosphorylation and modulation of a kainate receptor (GluR6) by cAMP-dependent protein kinase. Science 259:1173-1175.

Wilding TJ, Huettner JE (1996) Antagonist pharmacology of kainateand $\alpha$-amino-3-hydroxy-5-methyl-4-isoxazolepropionic acid-preferring receptors. Mol Pharmacol 49:540-546.

Wo ZG, Oswald RE (1994) Transmembrane topology of two kainate receptor subunits revealed by N-glycosylation. Proc Natl Acad Sci USA 91:7154-7158.

Wood MW, VanDongen HMA, VanDongen AMJ (1995) Structural conservation of ion conduction pathways in $\mathrm{K}$ channels and glutamate receptors. Proc Natl Acad Sci USA 92:4882-4886.

Yakel JL, Vissavajihala P, Derkach VA, Brickey DA, Soderling TR (1995) Identification of a $\mathrm{Ca}^{2+} /$ calmodulin-dependent protein kinase II regulatory phosphorylation site in non- $N$-methyl-D-aspartate glutamate receptors. Proc Natl Acad Sci USA 92:1376-1380. 